\title{
Modelo de suplementação nutricional com fatores hepatotróficos aumenta proliferação celular em fígado de ratos sadios
}

[Model of nutritional supplementation with hepatotrophic factors increases cell proliferation in the liver of healthy rats]

T.P.A. Aloia ${ }^{1}$, B. Cogliati $^{1}$, R.R. Guerra ${ }^{1}$, O.M. Parra ${ }^{2}$, M.L.Z. Dagli ${ }^{1}$, F.J. Hernandez-Blazquez ${ }^{1}$

${ }^{1}$ Faculdade de Medicina Veterinária e Zootecnia - USP

Av. Prof. Dr. Orlando de Paiva, 87

05508-900 - São Paulo, SP

${ }^{2}$ Faculdade de Medicina - USP - São Paulo, SP

\begin{abstract}
RESUMO
Foram avaliados dois protocolos de administração, em ratos sadios, de uma solução de fatores hepatotróficos (FH), composta por aminoácidos, vitaminas, sais minerais, glicose, insulina, glucagon e triiodotironina $\left(\mathrm{T}_{3}\right)$. A solução foi administrada durante 10 dias, $40 \mathrm{mg} / \mathrm{kg} / \mathrm{dia}$, i.p., em duas, grupo $2 \mathrm{xFH}(\mathrm{n}=15)$, ou três doses, grupo $3 x F H(n=15)$, diárias. Foram observados os efeitos na proliferação celular dos hepatócitos, na angiogênese e na matriz extracelular hepática, assim como as possíveis reações adversas. Os animais dos grupos $2 \mathrm{xFH}$ e $3 \mathrm{xFH}$ apresentaram aumento da massa hepática de $30,1 \%$ e $22,5 \%$, respectivamente, em relação ao grupo-controle (CT; n=15). O índice de proliferação hepatocelular foi maior nos grupos $2 x F H(1,4 \%)$ e $3 x F H(1,2 \%)$ em relação ao grupo CT $(0,53 \%)$, e a densitometria relativa do fator de crescimento do endotélio vascular pelo imunoblot não revelou diferença estatística entre os três grupos. Nos grupos $2 \mathrm{xFH}$ e $3 \mathrm{xFH}$, houve redução do colágeno intersticial em relação ao grupo CT. A solução de FH estimulou o crescimento hepático e reduziu o volume de colágeno perissinusoidal. A administração em três doses diárias resultou em mortalidade de $26,7 \%$, possivelmente pelo excessivo estresse da manipulação e pela menor adaptação fisiológica dos ratos, o que não ocorreu nos grupos 2xFH e CT. Para esse tipo de abordagem em ratos, o procedimento experimental mais apropriado, seguro, com melhor chance de adaptação dos animais e com resultados significativos é a aplicação dos FH em duas doses diárias.
\end{abstract}

Palavras-chave: rato, colágeno, fator hepatotrófico, modelo experimental, nutrição parenteral

\begin{abstract}
Two protocols of hepatotrophic factors $(H F)$ administration, in solution composed by aminoacids, vitamins, mineral salts, glucose, insulin, glucagon, and triiodothyronine were evaluated in healthy rats. This solution was administered for 10 days, (40mg/kg/day) i.p., in two (group $2 x F H ; n=15)$ or three daily doses (group $3 x F H n=15)$. The effects on hepatocytes cell proliferation, angiogenesis, and hepatic extracellular matrix, and also possible adverse reactions were analyzed. Animals of groups $2 x F H$ and $3 x F H$ presented an increase in hepatic mass of $30.1 \%$ and $22.5 \%$, respectively, when compared rats of control group $(C T ; n=15)$. Hepatocellular proliferation index was higher in rats of groups $2 x F H(1.4 \%)$ and $3 x F H(1.2 \%)$ when compared to CT group animals $(0.53 \%)$, and the relative densitometry of the vascular endothelial growth factor analyzed with immunoblot did not show a significant difference among the three groups. Rats of groups $2 x F H$ and $3 x F H$ showed a reduction of interstitial collagen when compared to CT rats. HF solution stimulated hepatic growth and reduced the volume of perisinusoidal collagen. Administration in three daily doses resulted in $26.7 \%$ mortality, possibly due to excessive stress from manipulation and lower physiological adaptation of rats, which did not occur in rats of groups $2 x F H$ and $C T$. The more appropriate and safer experimental procedure for this approach in rats with higher chance of animal adaptation and significant results is the application of HF in two daily doses.
\end{abstract}

Keywords: rat, collagen, hepatotrophic factors, experimental model, parenteral nutrition

Recebido em 20 de novembro de 2009

Aceito em 2 de agosto de 2010

E-mail: aloiathi@usp.br 


\section{INTRODUÇÃO}

Diversos fatores de crescimento e citocinas estão envolvidos no processo de regeneração hepática, porém fatores nutricionais também atuam modificando ou acionando respostas proliferativas (Holecek, 1999; Fausto et al., 2006). Sabe-se que animais desnutridos apresentam um retardo no processo regenerativo do fígado (Skullman et al., 1994). Assim, a suplementação nutricional torna-se uma estratégia terapêutica de grande potencial clínico.

$\mathrm{Na}$ prática, um suporte nutricional adequado promove melhor qualidade de vida ao paciente, podendo ser usado também como adjuvante para o aumento da massa hepática nos casos de transplantes intervivos (Nompleggi e Bonkovsky, 1994). Pacientes cirróticos também necessitam de uma intensiva suplementação nutricional, uma vez que pacientes desnutridos apresentam um quadro mais acentuado de lesão hepática e de outros sintomas decorrentes (O’Brien e Williams, 2008).

O mecanismo de absorção dos nutrientes devese, principalmente, ao endotélio fenestrado dos sinusoides, que expõe os hepatócitos diretamente a uma série de fatores hepatotróficos (Michalopoulus e Defrances, 1997). Dessa maneira, o suporte vascular é essencial para o suprimento nutricional e o trofismo celular. $\mathrm{Da}$ mesma forma, $\mathrm{o}$ fator de crescimento do endotélio vascular (VEGF) estimula a proliferação e a migração das células sinusoidais (Shimizu et al., 2001b) e possui uma importante contribuição no processo regenerativo hepático.

O sinergismo entre nutrientes e fatores de crescimento representa um importante estímulo na proliferação celular (Mazza-Stalder e Sauty, 2003), sugerindo que nenhuma substância isolada seria suficiente para regular todo o processo regenerativo no fígado (Ramalho et al., 1993; Fladmark et al., 1997). Nesse contexto, Parra et al. (1992; 1994; 1995; 1996) propuseram o uso de uma solução nutricional de fatores hepatotróficos $(\mathrm{FH})$ para auxiliar a regeneração hepática. Essa solução é composta por diversos aminoácidos e vitaminas, sais minerais, glicose, insulina, glucagon e tri-iodotironina $\left(\mathrm{T}_{3}\right)$. A insulina e o glucagon são importantes para o metabolismo dos hepatócitos (Jesus et al., 2000), enquanto $\mathrm{o}_{3} \mathrm{~T}_{3}$ atua como mitógeno primário, potencializando a proliferação celular em fígados hepatectomizados (Malik et al., 2003; 2005).

Parra et al. (1992; 1994) demonstraram aumento do volume hepático e redução do colágeno perissinusoidal em fígado de ratos sadios após a administração intraperitoneal (i.p.) desta solução de FH. Entretanto, os autores relataram alta taxa de mortalidade dos animais, provavelmente ocasionada pelo grande influxo de nutrientes em apenas uma aplicação. Desde então, outros trabalhos surgiram, apresentando resultados com aplicação terapêutica em doenças hepáticas crônicas e como importante adjuvante em hepatectomias parciais (Parra et al., 1995; 1996).

Portanto, devido ao potencial terapêutico e clínico da solução de $\mathrm{FH}$, este trabalho teve o objetivo de propor uma adequação do protocolo experimental em ratos para a redução da mortalidade. Além disso, avaliou-se o efeito dessa solução de fatores hepatotróficos na proliferação celular, na proporção volumétrica de colágeno e em possíveis mecanismos angiogênicos no tecido hepático.

\section{MATERIAL E MÉTODOS}

Foram utilizadas 45 ratas Wistar fêmeas pesando aproximadamente $200 \pm 20 \mathrm{~g}$ e com dois meses de idade. Os animais, alojados em gaiolas em grupos de cinco, em ambiente com arcondicionado, exaustor e fotoperíodo natural, receberam ração à vontade e água ad libitum. Todos os animais receberam tratamento de acordo com os critérios do Guia de Cuidados e Uso de Animais de Laboratório (ILAR, 1996). O experimento foi aprovado pelo Comitê de Bioética da Faculdade de Medicina Veterinária e Zootecnia da Universidade de São Paulo, número de bioética 804/2005.

Os animais foram distribuídos em três grupos experimentais: grupo $2 x \mathrm{xH} \quad(\mathrm{n}=15)$, que recebeu duas doses diárias da solução de fatores hepatotróficos, $20 \mathrm{~mL} / \mathrm{kg} / \mathrm{dose}$, intraperitonealmente, durante 10 dias; grupo $3 \mathrm{xFH}(\mathrm{n}=15)$, que recebeu três doses diárias da mesma solução de $\mathrm{FH}, 13,3 \mathrm{~mL} / \mathrm{kg} /$ dose, intraperitonealmente, durante 10 dias; e grupo CT $(n=15)$, que foi tratado com solução salina $(0,9 \%)$ nas mesmas condições dos grupos tratados com FH. A solução de $\mathrm{FH}$ continha glicose $(104 \mathrm{~g})$, solução de aminoácidos $(200 \mathrm{~mL}$ 
- ver mais adiante), piridoxina ( $2 \mathrm{mg}$ ), pantonato de cálcio $(2 \mathrm{mg})$, tiamina $(30 \mathrm{mg})$, fosfato de riboflavina (4mg), cloridrato de potássio $(1,43 \mathrm{~g})$, bicarbonato de sódio $(1,5 \mathrm{~g})$, nicotinamida (50mg), fosfato de monopotássio (750mg), sulfato de magnésio (500mg), vitamina C (500mg), insulina $(62,5 \mathrm{U})$, glucagon $(0,625 \mathrm{mg})$, ácido fólico $(2,5 \mathrm{mg})$, vitamina B12 $(31,25 \mu \mathrm{g})$, sulfato de zinco $(3,125 \mathrm{mg})$ e água destilada (500mL). A solução de aminoácidos continha: Lfenilalanina (1,080mg), L-isoleucina (740mg), Lleucina (1,960mg), L-acetato de lisina (1,180mg), L-metionina (1,060mg), L-treonina (980mg), L-triptofano (360mg), L-valina $(1,060 \mathrm{mg})$, L-arginina base $(1,060 \mathrm{mg})$, Lhistidina base $(920 \mathrm{mg})$, L-alanina $(2,060 \mathrm{mg})$, Lasparginina (790mg), L-ácido aspártico (540mg), L-ácio glutâmico (500mg), L-cisteína (30mg), Lornitina $(520 \mathrm{mg})$, L-prolina $(1,680 \mathrm{mg})$, L-serina (500mg), L-tirosina $(320 \mathrm{mg})$, glicina $(1,600 \mathrm{mg})$ em $200 \mathrm{~mL}$ de água destilada. O hormônio Ltriiodotironina $\left(\mathrm{T}_{3}\right)$ foi diluído $(2,26 \mu \mathrm{g} / 200 \mathrm{~g}$ de peso corpóreo do rato/dia) em solução alcoólica $(1 \mathrm{mg} / 10 \mathrm{~mL})$ e acrescentado na solução de FH antes das aplicações.

Os animais foram sacrificados por excessiva dose de isufluorano, seguido da retirada dos órgãos e mensuração dos parâmetros biométricos. Foram mensurados o peso dos ratos no início (PRi) e no final (PRf) do experimento, o peso da massa do fígado (MF), o índice hepatossomático (IHS) [(MF.100)/PRf], o ganho de peso (GP) [(PRf-PRi).100/PRi], o volume do fígado (VF) pelo método de Scherle (Scherle, 1970), densidade do fígado (DF) (MF/VF), o peso das vísceras (VIS), o índice das vísceras/peso total do animal (IVP) [(VIS.100)/PRf], o peso da carcaça (CAR) e o índice do fígado/carcaça (IFC) [(MF.100)/CAR].

Amostras de fígado, rins, coração, pulmões e baço, coletadas de todos os animais para análise histológica, foram fixadas em solução de Methacarn por 24 horas e emblocadas em parafina. As lâminas foram coradas por hematoxilina-eosina (HE) e picrossírius tratado com ácido fosfomolíbdico $2 \%$ modificado (Puchtler e Isler, 1958).

Cortes de $5 \mu \mathrm{m}$ foram imersos em tampão citrato $(\mathrm{pH} 6,0)$ e aquecidos por micro-ondas $(700$ watts) durante 12 minutos. A peroxidase endógena foi bloqueada pela imersão das lâminas em solução de $\mathrm{H}_{2} \mathrm{O}_{2} 5 \%$ diluída em metanol por 30 minutos. Após lavagem em PBS (pH 7,2), as reações inespecíficas foram bloqueadas pelo leite em pó desnatado (Itambé - Belo Horizonte) por 20 minutos a $37^{\circ} \mathrm{C}$; os cortes histológicos foram lavados novamente e incubados por 12 horas a $4^{\circ} \mathrm{C}$ com anticorpo monoclonal de camundongo anti-PCNA clone PC10 (Dako, Carpinteria, CA, USA, M0879) diluído em PBS (1:100). Em seguida, os cortes foram incubados com anticorpo secundário biotinilado por 30 minutos, lavados em PBS e incubados com o complexo estreptavidina-peroxidase (LSAB - Dako, Carpinteria, CA, USA) por 30 minutos. As células positivas foram evidenciadas por meio da revelação dos cortes pelo DAB (3'3Diaminobenzidine Tetrahydrochloride, Dako, Carpinteria, CA, USA). O índice de proliferação celular foi calculado pela porcentagem de células PCNA-positivas em 1000 hepatócitos.

A densidade volumétrica das fibras colágenas nos espaços perissinusoidais foi quantificada em cada foto usando-se o software KS 400.3 Zeiss ${ }^{\circledR}$. As lâminas coradas por picrossírius tratado com ácido fosfomolíbdico $2 \%$ foram digitalizadas por meio da câmera Axio CAM HRC Zeiss ${ }^{\circledR}$, e a área ocupada pela fibra colágena foi mensurada em relação ao total da área fotografada. Os resultados foram expressos pela proporção volumétrica de colágeno (média \pm desviopadrão), considerando-se a área total do campo fotografado $\left(67.014,21 \mu \mathrm{m}^{2}\right)$.

A proteína total foi extraída de fragmentos congelados de fígado. Os fragmentos foram homogeneizados em tampão contendo $60 \mathrm{mM}$ Tris- $\mathrm{HCl}, \mathrm{pH} 6,8, \mathrm{SDS}$ a $2 \%$, glicerol a $12 \%$, ditiotreitol $0,1 \mathrm{M}$ e fluoreto de fenilmetilsulfonil (PMSF) 1mM. A concentração de proteínas nesse homogenato foi determinada utilizando-se o kit da Bio-Rad ${ }^{\hat{a}}$, e a leitura realizada em espectrofotômetro a $595 \mathrm{~nm}$. As proteínas foram resolvidas em eletroforese num gel de poliacrilamida a $12 \%$ em dodecil sulfato de sódio (SDS) e transferidas durante 45 minutos, a $45 \mathrm{~mA}$, para membranas de difluoreto de polivinilideno (PDVF). As membranas foram incubadas com anticorpo monoclonal anti-VEGF (1:200; Santa Cruz), diluído em solução de PBS contendo $5 \%$ de leite desnatado por 2 horas, em temperatura ambiente. Em seguida, a membrana foi incubada com anticorpo secundário de cabra 
anticamundongo conjugado com peroxidase (1:500 em 5\% de skim milk; Zymed Laboratories, San Francisco, EUA) por duas horas, em temperatura ambiente. A reação foi revelada com uma solução contendo diaminobenzidina (DAB, Sigma Aldrich), sulfato de níquel e peróxido de hidrogênio. $\mathrm{O}$ escore da intensidade de marcação foi quantificado pelo sistema Image Master (Amersham Pharmacia Biotech, Little Chalfont, UK). A intensidade do escore do VEGF foi analisada pela comparação do volume da banda com o controle endógeno beta-actina.
Análise de variância e testes Tukey e t-Student foram aplicados aos grupos quando pertinente, considerando-se $\mathrm{P}<0,05$.

\section{RESULTADOS}

Os animais do grupo $3 \mathrm{xFH}$ apresentaram índice de mortalidade de 26,7\%, mais alto que o dos animais dos grupos $2 \mathrm{xFH}$ e controle, e nestes não houve mortalidade durante o procedimento experimental. Os parâmetros somáticos e viscerais analisados foram diferentes $(\mathrm{P}<0,05)$ entre os grupos tratados com a solução de $\mathrm{FH}$ e o grupo-controle (Tab. 1), e não houve diferença entre os grupos $2 \mathrm{xFH}$ e $3 \mathrm{xFH}(\mathrm{P}>0,05)$.

Tabela 1 Parâmetros de massa e volume no grupo de ratos controle (CT) ou tratados com solução de fatores hepatotróficos por duas vezes ao dia $(2 \mathrm{xFH})$ ou três vezes ao dia $(3 \mathrm{xFH})$

\begin{tabular}{|c|c|c|c|c|c|c|c|c|c|c|c|c|}
\hline Grupo & $\mathrm{N}$ & $\begin{array}{l}\text { PRi } \\
(\mathrm{g})\end{array}$ & $\begin{array}{l}\text { PRf } \\
(g)\end{array}$ & $\begin{array}{l}\text { GP } \\
(\mathrm{g})\end{array}$ & $\begin{array}{l}\text { MF } \\
(\mathrm{g})\end{array}$ & $\begin{array}{l}\text { IHS } \\
(\%)\end{array}$ & $\begin{array}{c}\mathrm{VF} \\
\left(\mathrm{cm}^{3}\right)\end{array}$ & $\begin{array}{c}\text { DF } \\
\left(\mathrm{cm}^{3} / \mathrm{g}\right)\end{array}$ & $\begin{array}{l}\text { VIS } \\
(\mathrm{g})\end{array}$ & $\begin{array}{l}\text { IVP } \\
(\%)\end{array}$ & $\begin{array}{c}\text { CAR } \\
(\mathrm{g})\end{array}$ & $\begin{array}{l}\text { IFC } \\
(\%)\end{array}$ \\
\hline $\mathrm{CT}$ & 15 & $\begin{array}{l}211,6 \\
(14,7)\end{array}$ & $\begin{array}{l}212,3 \\
(13,6)\end{array}$ & $\begin{array}{l}0,7 a \\
(3,1)\end{array}$ & $\begin{array}{l}9,3 a \\
(1,0)\end{array}$ & $\begin{array}{l}4,4 a \\
(0,4)\end{array}$ & $\begin{array}{l}8,6 a \\
(0,9)\end{array}$ & $\begin{array}{l}1,08 \mathrm{a} \\
(0,01)\end{array}$ & $\begin{array}{l}46,0 \mathrm{a} \\
(3,8)\end{array}$ & $\begin{array}{c}21,6 \mathrm{a} \\
(1,1)\end{array}$ & $\begin{array}{c}166,3 a \\
(10,8)\end{array}$ & $\begin{array}{l}5,6 a \\
(0,6)\end{array}$ \\
\hline $2 \mathrm{xFH}$ & 15 & $\begin{array}{l}220,4 \\
(11,6)\end{array}$ & $\begin{array}{l}234,7 \\
(13,9)\end{array}$ & $\begin{array}{c}14,3 b \\
(5,9)\end{array}$ & $\begin{array}{l}12,1 \mathrm{~b} \\
(1,2)\end{array}$ & $\begin{array}{l}5,2 b \\
(0,4)\end{array}$ & $\begin{array}{c}11,3 b \\
(1,1)\end{array}$ & $\begin{array}{l}1,06 b \\
(0,01)\end{array}$ & $\begin{array}{c}52,2 b \\
(3,9)\end{array}$ & $\begin{array}{c}22,2 \mathrm{a}, \mathrm{b} \\
(1,2)\end{array}$ & $\begin{array}{c}182,5 b \\
(11,6)\end{array}$ & $\begin{array}{l}6,6 \mathrm{~b} \\
(0,6)\end{array}$ \\
\hline $3 \times F H$ & 13 & $\begin{array}{l}198,2 \\
(7,5)\end{array}$ & $\begin{array}{l}215,1 \\
(15,7)\end{array}$ & $\begin{array}{l}16,9 b \\
(15,0)\end{array}$ & $\begin{array}{l}11,4 b \\
(1,2)\end{array}$ & $\begin{array}{l}5,3 b \\
(0,4)\end{array}$ & $\begin{array}{c}10,6 b \\
(1,1)\end{array}$ & $\begin{array}{c}1,07 a, b \\
(0,01)\end{array}$ & $\begin{array}{c}50,8 \mathrm{a}, \mathrm{b} \\
(3,8)\end{array}$ & $\begin{array}{c}23,5 b \\
(2,1)\end{array}$ & $\begin{array}{c}164,3 \mathrm{a} \\
(11,9)\end{array}$ & $\begin{array}{l}6,9 \mathrm{~b} \\
(0,7)\end{array}$ \\
\hline $\begin{array}{c}\text { Relação } \\
\text { CT- } \\
2 x F H \\
(\%) \\
\end{array}$ & I & I & I & $+6,4$ & $+30,1$ & $+18,1$ & $+31,3$ & $-1,8$ & $+13,4$ & NS & $+9,7$ & $+17,8$ \\
\hline $\begin{array}{c}\text { Relação } \\
\text { CT- } \\
3 \times \text { FH } \\
(\%) \\
\end{array}$ & I & I & I & $+8,5$ & $+22,5$ & $+20,4$ & $+23,2$ & NS & NS & $+8,7$ & NS & $+23,3$ \\
\hline $\begin{array}{c}\text { Relação } \\
2 \times{ }^{x}{ }^{2}- \\
3 x F H \\
(\%)\end{array}$ & I & I & I & NS & NS & NS & NS & NS & NS & NS & $-1,2$ & NS \\
\hline
\end{tabular}

*Médias na mesma coluna seguidas por letras diferentes indicam diferenças entre valores, segundo o teste Tukey $(\mathrm{P} \leq 0,05)$. Desvio-padrão indicado entre parênteses.

PRi - peso do rato inicial; PRf - peso do rato final; GP - ganho de peso; MF - massa do fígado; IHS índice hepatossomático; VF - volume do fígado; DF - densidade do fígado; VIS - peso das vísceras; IVP - índice vísceras-peso total do animal; CAR - peso da carcaça, IFC - índice fígado-carcaça, NS diferença estatística entre as médias não significativa.

Os pulmões, os rins, o coração e o baço dos animais tratados com a solução de FH não apresentaram lesões. $\mathrm{O}$ índice de proliferação celular (PCNA) foi maior nos animais tratados com a solução de FH quando comparado aos do grupo CT, com aumento de $1,4 \%$ no grupo $2 \mathrm{xFH}, 1,2 \%$ no grupo $3 \mathrm{xFH}$ e $0,53 \%$ no grupo $\mathrm{CT}(\mathrm{P}<0,05)$. Não houve diferença significativa entre os grupos tratados com as diferentes doses de FH (P>0,05; Fig. 1A, C e E). 

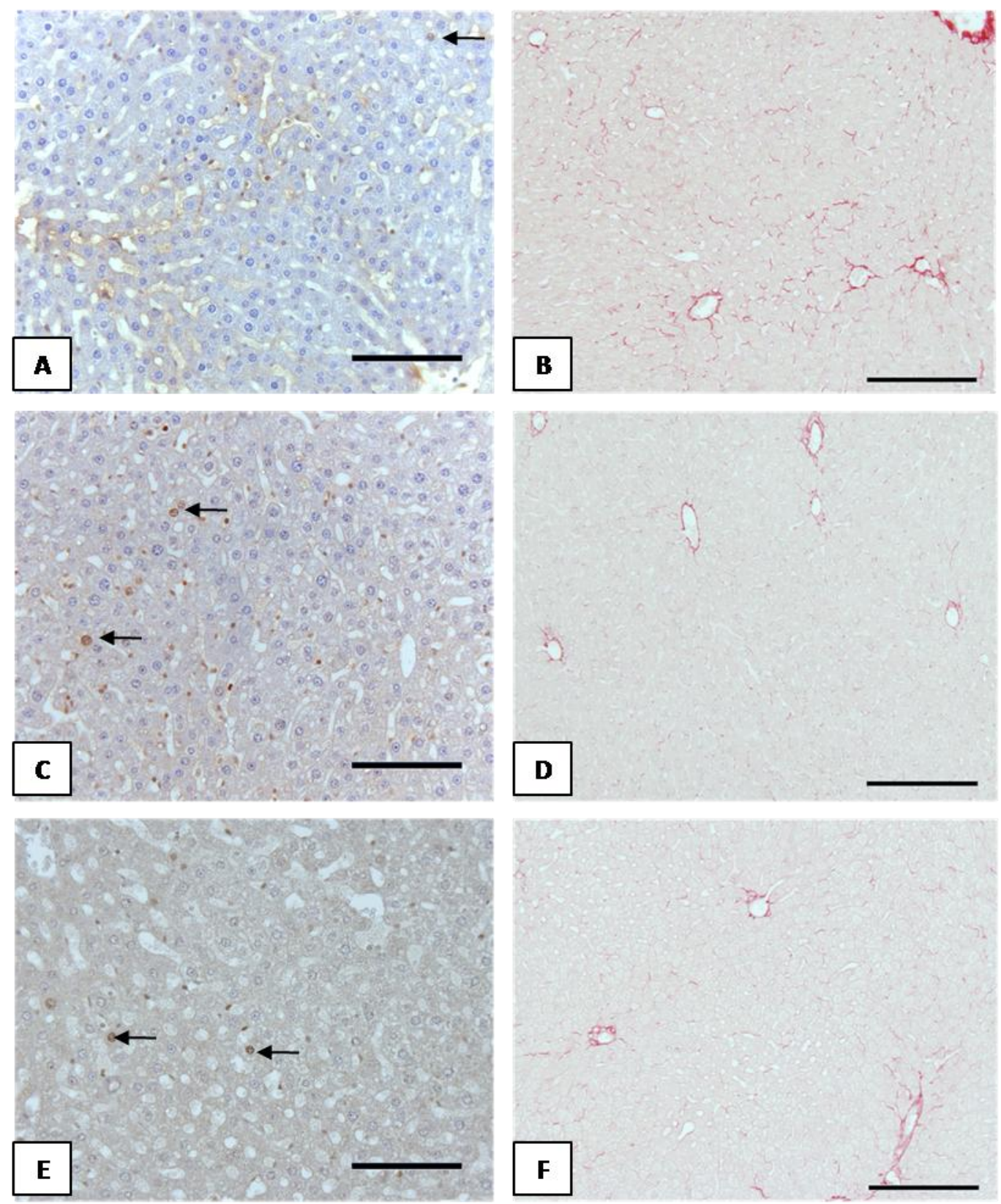

Figura 1. Fígado de rato. A, C e E: fotomicrografias mostrando hepatócitos marcados positivamente na reação imunoistoquímica para anti-PCNA. B, D, F: fotomicrografias evidenciando fibras colágenas coradas pela técnica de picrossírius tratado com ácido fosfomolíbdico $2 \%$ (A e B: grupo-controle que recebeu solução salina $0,9 \%$; C e D: grupo que recebeu duas doses de solução de fatores hepatotróficos e E e F: grupo que recebeu três doses de solução de fatores hepatotróficos). Barra de escala de A, C e E = $100 \mu \mathrm{m}$; Barra de escala de B, D, F = 200 $\mu \mathrm{m}$. Setas indicam marcação positiva para PCNA. 


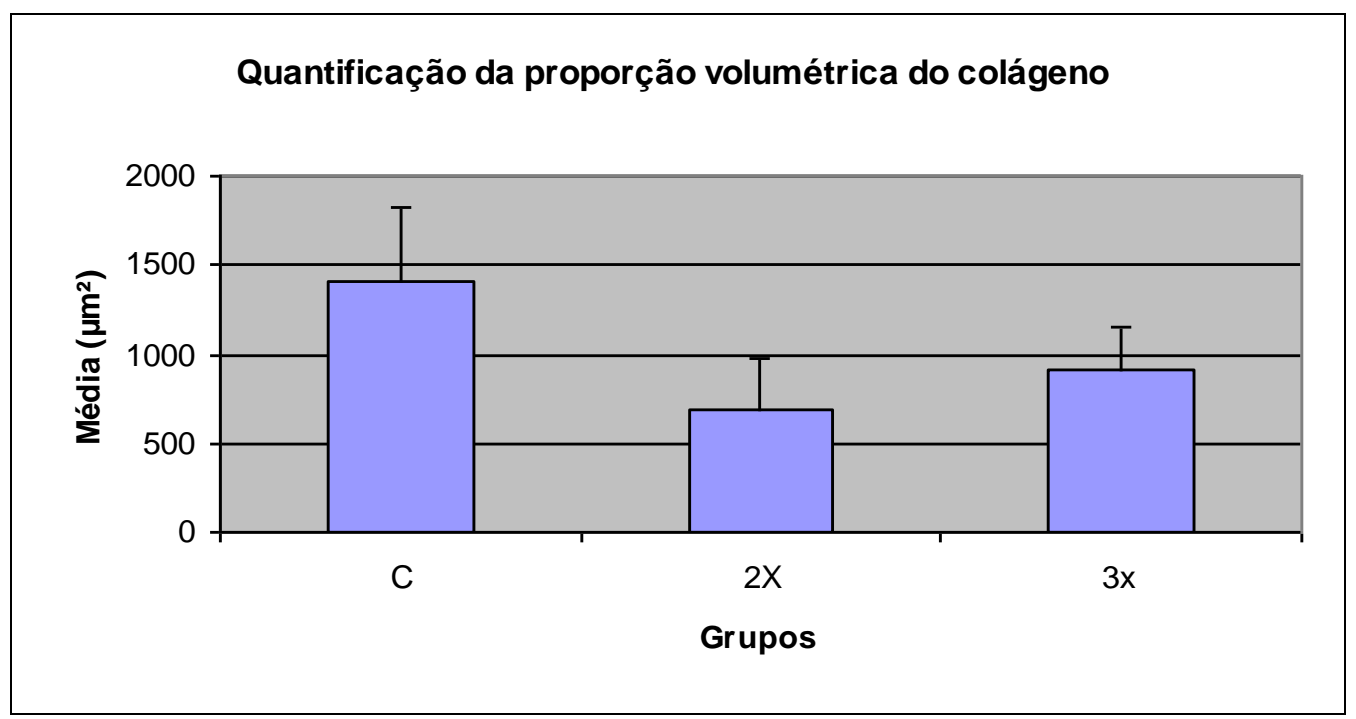

Figura 2. Colágeno perissinusoidal do fígado dos ratos. A coluna do grupo-controle apresenta diferença estatística quando comparada às dos grupos que receberam duas doses (2x) ou três doses (3x) da solução de fatores hepatotróficos $(\mathrm{P}<0,05)$.

Houve redução significativa na proporção volumétrica de colágeno perissinusoidal no fígado de animais dos grupos tratados com a solução de fatores hepatotróficos $(\mathrm{P}<0,05)$. Os animais do grupo $2 \mathrm{xFH}$ apresentaram redução de $51,8 \%$ na proporção volumétrica de colágeno em relação aos do grupo CT, enquanto nos animais do grupo $3 \mathrm{xFH}$ a redução foi de $34,9 \%$. Entre os dois grupos tratados com a solução de $\mathrm{FH}$, não houve diferença significativa na proporção volumétrica de colágeno $(\mathrm{P}>0,05$; Fig. $1 \mathrm{~B}, \mathrm{D}$ e F; Fig. 2).

A densitometria relativa do imunoblot para VEGF e $\beta$-actina não apresentou diferença entre os três grupos estudados (Fig. 3; P>0,05).

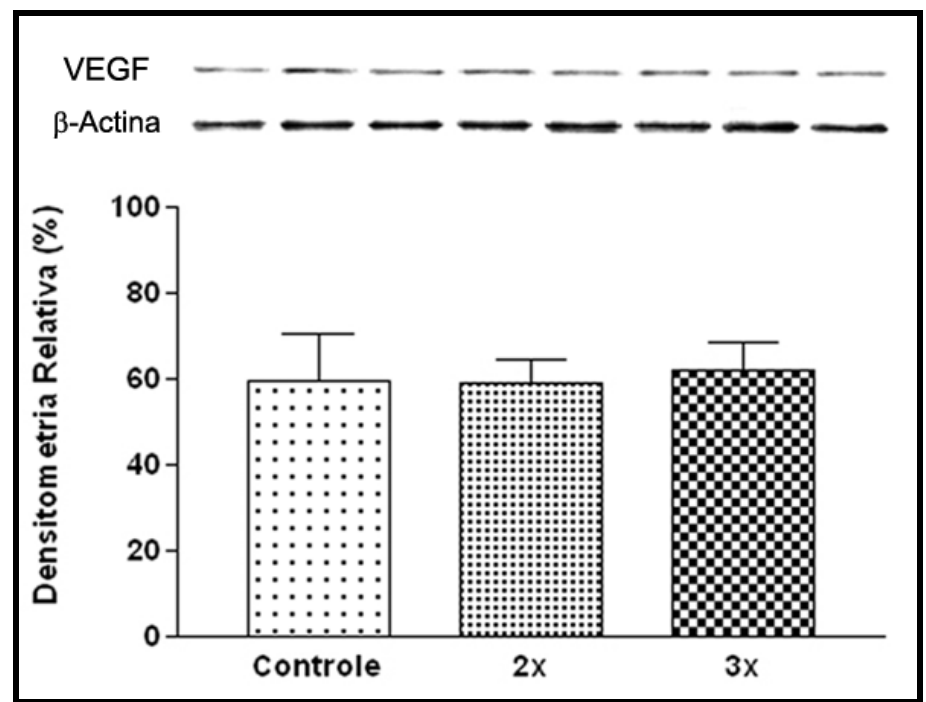

Figura 3. Detecção por Western Blotting do fator de crescimento do endotélio vascular (VEGF) nas amostras de tecido hepático de ratos. 


\section{DISCUSSÃO}

A composição da solução de fatores hepatotróficos utilizada por Parra et al. (1992; $1994 ; 1995$; 1996) possui a finalidade de simular a ação de nutrientes e de fatores de crescimento que ascendem ao fígado pela veia porta. $\mathrm{O}$ uso da via intraperitonial para a administração dessa solução baseia-se no pressuposto de que a absorção da maior parcela dessas substâncias ocorre, principalmente, através dos vasos do peritônio, fluindo para sistema porta e sinusoides hepáticos. Desta forma, a barreira representada pela mucosa do aparelho digestório nas administrações orais seria eliminada, promovendo um melhor suporte nutricional aos pacientes debilitados (Parra et al., 1982; Matsuda et al., 1997).

A suplementação nutricional possui crescente importância no tratamento de doenças hepáticas (O’Brien e Williams, 2008), melhorando o estado imunológico e a função hepática desses pacientes. $\mathrm{O}$ fornecimento adequado de proteínas, vitaminas e sais minerais demonstra ser essencial para a correção da desnutrição proteica e desidratação, auxiliando na síntese de proteínas e na regeneração hepática (Rombeau e Rolandelli, 2004). Além dos principais nutrientes envolvidos na recuperação desses pacientes, alguns hormônios também exercem importante função no metabolismo e trofismo hepático, como a insulina, o glucagon e o $\mathrm{T}_{3}$ (AnkomaSey, 1999; Jesus et al., 2000).

Os animais do grupo $2 \mathrm{xFH}$ apresentaram acréscimo de $30,1 \%$ na massa hepática, enquanto os animais do grupo $3 \mathrm{xFH}$ obtiveram aumento de $22,5 \%$ em relação ao grupo-controle. Nesse parâmetro, também não houve diferença estatística entre os tratamentos. No entanto, o índice de mortalidade dos animais do grupo $3 \mathrm{xFH}$ foi $26,7 \%$ mais alto que nos dos grupos $2 \mathrm{xFH}$ e controle. Nestes não houve mortalidade. Dessa maneira, os animais que receberam três injeções diárias apresentaram maiores problemas relacionados à adaptação, ao estresse e à metabolização dos nutrientes administrados intraperitonealmente. Os dados relacionados ao crescimento hepático foram semelhantes aos descritos por Parra et al. (1992; 1996), que obtiveram aumento entre 34,5 e $121 \%$ em animais que receberam uma dose única da solução de FH (40mg/kg), porém com alto índice de mortalidade.

Estudos in vitro evidenciaram que os mecanismos proliferativos dos hepatócitos são regulados, principalmente, por mecanismos autócrinos a partir da liberação de fatores de crescimento (Mullhaupt et al., 1994). Como os animais tratados com a solução de $\mathrm{FH}$ apresentaram aumento de massa hepática em relação aos animais controles, isto sugere que houve hiperplasia ou hipertrofia celular, ocasionando o aumento de peso. No entanto, a avaliação da proliferação celular pela imunoistoquímica para PCNA comprovou que houve um processo de hiperplasia, com aumento na taxa de proliferação dos hepatócitos.

O expressivo aumento de massa hepática observado por Parra et al. (1992; 1994; 1995; 1996) em animais tratados com $\mathrm{FH}$ pode ter várias aplicações, como estimular o crescimento do fígado antes ou depois do transplante intervivo, acelerar o processo de regeneração hepática após hepatectomia parcial (Cogliati et al., 2004; Miranda et al., 2005), ou mesmo diminuir o colágeno em fígados com fibrose ou cirróticos (Pereira et al., 2003; HernandezBlazquez et al., 2005). No entanto, a aplicação dessa solução de FH necessita da padronização de um modelo experimental reprodutível, fidedigno e que permita a visualização dos principais resultados esperados pela terapia. Além disso, faz-se necessária a avaliação completa do animal que permitia a identificação de possíveis efeitos colaterais durante a terapia experimental. Nesse contexto, a aplicação da solução de $\mathrm{FH}$ nos grupos $2 \mathrm{xFH}$ e $3 \mathrm{xFH}$ não ocasionou alterações histológicas nos demais órgãos analisados, indicando baixa toxicidade.

O fígado dos animais tratados apresentou alterações significativas em relação à proporção volumétrica de colágeno (PVC). Os animais do grupo 2xFH apresentaram redução de $51,8 \%$ na PVC perissinusoidal em relação aos do grupocontrole. Os do grupo $3 \mathrm{xFH}$ apresentaram redução de 34,9\%. Não houve diferença significativa na PVC entre os grupos $2 \mathrm{xFH}^{\mathrm{e}}$ $3 \mathrm{xFH}$, indicando que ambos os tratamentos foram eficazes na redução do colágeno perissinusoidal. Estes dados foram semelhantes aos obtidos por Parra et al. (1996), em que foi demonstrada redução de $37,46 \%$ na PVC em 
animais tratados com uma injeção diária única de FH $(40 \mathrm{mg} / \mathrm{kg})$, durante sete dias. No entanto, os autores demonstraram que a utilização dessa dosagem única promovia alta taxa de mortalidade dos animais $(60 \%)$, principalmente devido ao rápido crescimento do fígado e sobrecarga de nutrientes (Parra et al., 1995).

A formação dos sinusoides hepáticos tem importante papel para o suprimento de sangue nos locais onde células hepáticas estão proliferando (Arai et al., 1995; Shimizu, et al., 2001a). No fígado, o fator de crescimento do endotélio vascular (VEGF) é secretado pelos hepatócitos, e estudos já comprovaram sua ação no tecido hepático com atividade regenerativa (Shimizu et al., 2001b). No entanto, a quantificação relativa de VEGF do tecido hepático no imunoblot não demonstrou aumento significativo entre os grupos $2 \mathrm{xFH}, 3 \mathrm{xFH}$ e CT após 10 dias de tratamento com os $\mathrm{FH}$.

\section{CONCLUSÕES}

Os tratamentos com a solução de fatores hepatotróficos, fracionada em duas ou três aplicações, produziram resultados satisfatórios quanto ao crescimento hepático e à redução do colágeno perissinusoidal. $\mathrm{O}$ protocolo de duas doses diárias revelou-se melhor por não ter havido mortes. Assim, os animais tratados com duas doses diárias de FH forneceram melhor resposta fisiológica e adaptativa, pois, além de terem sido submetidos a menos condições de estresse, o período de ação dessa solução nutritiva demonstrou ser ideal nas condições metodológicas aplicadas, aumentando o volume hepático e a proliferação dos hepatócitos e reduzindo a densidade volumétrica de colágeno perissinusoidal.

\section{AGRADECIMENTOS}

Ao laboratório Fórmula Medicinal (São Paulo $\mathrm{SP})$, por fornecer a solução de fatores hepatotróficos durante o período experimental.

\section{REFERÊNCIAS BIBLIOGRÁFICAS}

ANKOMA-SEY, V. Hepatic regeneration-revisiting the myth of Prometheus. News Physiol. Sci., v.14, p.149-155, 1999.

ARAI, M.; MOCHIDA, S.; OHNO, A. et al. Blood coagulation equilibrium in rat liver microcirculation as evaluated by endothelial cell thrombomodulin and macrophage tissue factor. Thromb. Res., v.80, p.1131231995 .
COGLIATI, B.; PEREIRA, H.M.; PARRA, O.M. et al. Avaliação da proliferação celular hepática após a administração de fatores hepatotróficos em ratos hepatectomizados. In: SIMPÓSIO INTERNACIONAL DE INICIAÇÃO CIENTÍFICA DA USP, 12., 2004, São Paulo. Anais... São Paulo, $2004 . \quad$ Disponível em: <http://www.usp.br/siicusp/12osiicusp/ficha1116.htm >. Acessado em: 3 out. 2009

FAUSTO, N.; CAMPBELL, J.S.; KIMBERLY, J.R. Liver regeneration. Hepatology, v.43, p.45-53, 2006.

FLADMARK, K.E.; GJERTSEN, B.T.; MOLVEN, A. et al. Gap junctions and growth control in liver regeneration and in isolated rat hepatocytes. Hepatology, v.25, p.847-855, 1997.

HERNANDEZ-BLAZQUEZ, F.J.; GUERRA, R.R.; TROTTA, M. et al. Effect of hepatotrophics factors in rat liver cirrhosis. In: CONGRESO NACIONAL DE HISTOLOGIA E INGENIERIA TISULAR. FIRST INTERNATIONAL CONGRESS OF HISTOLOGY AND TISSUE ENGINEERING, 13., 2005, Alcalá de Henares. Proceedings... Alcalá de Henares, 2005. [Histology and Histopathology] v.S1, p.S156-S156.

HOLECEK, M. Nutritional modulation of liver regeneration by carbohyrates, lipids, and amino acids: a review. Nutrition, v.15, p.784-788, 1999.

GUIDE for the care and use of laboratory animals. ILAR, Washington: National Academy of Sciences, 1996.

JESUS, R.P.; WAITZBERG, D.L.; CAMPOS, F.G. Regeneração hepática: papel dos fatores de crescimento e nutrientes. Rev. Assoc. Med. Bras., v.46, p.242-254, 2000 .

MALIK, R.; MELLOR, N.; SELDEN, C. et al. Triiodothyronine enhances the regenerative capacity of the liver following partial hepatectomy. Liver Biol. Pathol., v.37, p.79-86, 2003.

MALIK, R.; HABIB, M.; TOOTLE, R. et al. Exogenous thyroid hormone induces liver enlargement, whilst maintaining regenerative potential - A study relevant to donor preconditioning. Am. J. Transplant., v.5, p.1801-1807, 2005.

MATSUDA, Y.; MATSUMOTO, K.; ICHIDA, T. et al. Preventive and therapeutic effects in rats of hepatocyte growth factor infusion on liver fibrosis/cirrhosis. Hepatology, v.215, p.81-89, 1997.

MAZZA-STALDER, J.; SAUTY, A. The hepatopulmonary syndrome [Le syndrome hépatopulmonaire (SHP). Med. Hyg., v.61, p.21892193, 2003.

MICHALOPOULUS, G.K.; DEFRANCES, M.C. Liver regeneration. Science, v.276, p.60-66, 1997. 
MIRANDA, G.P.; GUERRA, R.R.; PARRA, O.M. et al. Proliferação celular e aporte vascular na regeneração hepática estimulada por fatores hepatotróficos após hepatectomia parcial em ratos. In: SIMPÓSIO INTERNACIONAL DE INICIAÇÃO CIENTÍFICA DA USP, 13., 2005, São Paulo. Anais... São Paulo, 2005. Disponível em: <http://www.usp.br/siicusp/13osiicusp/aprovados/fich a440.htm>. $\quad$ Acessado em 3 out. 2009.

MULLHAUPT, B.; FEREN, A.; FODORE-JONES, A. et al. Liver expression of epidermal growth factor RNA. Rapid increases in immediate-early phase of liver regeneration. J. Biol. Chem., v.269, p.1966719670, 1994.

NOMPLEGGI, D.J.; BONKOVSKY, H.L. Nutritional supplementation in chronic liver disease: An analytical review. Hepatology, v.19, p.518-533, 1994.

O'BRIEN, A.; WILLIAMS, R. Rapid diagnosis of Wilson disease in acute liver failure: no more waiting for the ceruloplasmin level? Hepatology, v.48, p.10301032, 2008.

PARRA, O.M. Métodos de estímulo da regeneração do figado e perspectivas no tratamento da cirrose hepática. Revisão, comentários e contribuição. 1982. Dissertação (Mestrado) - Faculdade de Medicina da Universidade de São Paulo, São Paulo.

PARRA, O.M.; SAAD, W.A.; FERRAZ-NETO, J.BH.H. et al. Additional growth of an intact liver induced by exogenous hepatotrophic factors. A study in a rats. Arq. Bras. Cir. Dig., v.7, p.64-68, 1992.

PARRA, O.M.; SAAD, W.A.; SILVA, R.A.P.S. et al. Stimulation of intact rat liver by exogenous hepatotrophic factors with additional growth of its mass. Acta Cir. Bras., v.9, p.7-11, 1994.

PARRA, O.M.; SILVA, R.A.P.S.; SILVA, J.R.M. et al. Enhancement of liver size by stimulation of intact rat liver with exogenous hepatotrophic factors. São Paulo Med. J., v.113, p.941-947, 1995.
PARRA, O.M.; HERNANDEZ-BLASQUEZ, F.J.; SILVA, J.R.M. et al. Behavior of collagen extracellular liver matrix during regenerative growth after partial hepatectomy or after stimulation by exogenous hepatotrophic factors. Study in rats. Arq. Gastroenterol., v.33, p.212-216, 1996.

PEREIRA, H.M.; HERNADEZ-BLAZQUEZ, F.J.; PARRA, O.M. et al. Quantificação do tecido colágeno em fígado com fibrose induzida em ratas (Rattus norvegucus albinus) tratadas com fatores hepatotróficos exógenos. Arq. Cien. Vet. Zool. UNIPAR, v.6, p.196-217, 2003.

PUCHTLER, H.; ISLER, $H$. The effect of phosphomolybdic acid on the stainability of connective tissues by various dyes. J. Histochem. Cytochem., v.6, p.265,1958.

RAMALHO, F.S.; RAMALHO, L.N.Z.; ZUCOLOTO, S. et al. Regeneração hepática algumas definições num universo de incertezas. Acta Circ. Brasil, v.8, p.177-189, 1993.

ROMBEAU, J.L.; ROLANDELLI, R.H. Nutrição clínica: nutrição parenteral. 3.ed. São Paulo: Roca, 2004. p.576.

SCHERLE, W.A simple method for volumetry of organs in quantitative stereology. Mikroskopie, v.26, p.57-63, 1970 .

SHIMIZU, H.; MIYAZAKI, M.; ITO, H. et al. Mechanism of cold ischemia-reperfusion graft injury after orthotopic liver transplantation in rats. Hepatogastroenterology, v.48, p.216-219, 2001a.

SHIMIZU, H.; MIYAZAKI, M.; WAKABAYASHI, Y. et al. Vascular endothelial growth factor secreted by replicating hepatocytes induces sinusoidal endothelial cell proliferation during regeneration after partial hepatectomy in rats. J. Hepatol., v.34, p.683689, $2001 b$.

SKULLMAN, S.; WIREN, M.; GARLICK, P.J. et al. Protein synthesis in regenerating rat liver during malnutrition. J. Hepatol., v.21, p.174-181, 1994. 